\title{
Thrombocyte Alterations in Pregnant Women with Gestational Diabetes Mellitus
}

\author{
Senol SENTURK ${ }^{1}$, Mehmet KAGITCI ${ }^{2}$, Emine Seda GUVENDAG GUVEN ${ }^{3}$ \\ Rize, Turkey

\begin{abstract}
OBJECTIVE: Inflammation and its complications might develop in patients diagnosed with diabetes mellitus. It is shown that number, shape and functions of thrombocytes are important for development and prediction of inflammatory process. We aimed to show the changes of number, morphology and functions of platelets in the patients with gestational diabetes mellitus.
\end{abstract}

STUDY DESIGN: In a retrospective study, 928 cases in $24-28^{\text {th }}$ weeks of gestation followed regularly in the same clinic were included. We performed $75 \mathrm{~g}$ oral glucose tolerance test to all cases. Mean platelet volume, platelet distribution width and plateletcrit values of the cases with and without gestational diabetes mellitus according to American Diabetes Association criteria were compared to evaluate the platelet count, morphology, functions and activity.

RESULTS: Platelet count and plateletcrit values of cases were significantly higher in the patients with gestational diabetes mellitus. No significant differences were found between the cases with and without gestational diabetes mellitus in mean platelet volume and platelet distribution width values.

CONCLUSION: There is tendency to inflammation in gestational diabetes mellitus and platelets are part of inflamatuary process in human metabolism. Our findings show that, platelets are affected in gestational diabetes mellitus. We believe that further studies in this subject will help understanding of pathophysiology of gestational diabetes mellitus.

Keywords: Diabetes mellitus, Pregnancy, Gestational diabetes, Platelet activation

Gynecol Obstet Reprod Med 2018;24(1):12-16

\section{Introduction}

Many factors including obesity, sedentary lifestyle and environmental factors caused an increase in the frequency of type 2 diabetes mellitus (DM.) in recent years. DM. is an important health problem that might cause vascular problems

${ }^{1}$ Department of Gynecology and Obstetrics Recep Tayyip Erdogan University Medical School, Rize

${ }^{2}$ Department of Gynecology and Obstetrics Mersin State Hospital, Mersin

${ }^{3}$ Department of Obstetrics and Gynecology Karadeniz Technical University School of Medicine, Trabzon

Address of Correspondence: Mehmet Kagitci

Department of Gynecology and

Obstetrics, Mersin State Hospital,

33240 Mersin, Turkey

mehmetkagitci1@hotmail.com

Submitted for Publication: $\quad$ 16.05.2017

Accepted for Publication: $\quad$ 17.09.2017

\begin{tabular}{|c|c|}
\hline & Access this article online \\
\hline $\begin{array}{c}\text { Quick Response Code: } \\
\text { and }\end{array}$ & $\begin{array}{c}\text { Website: www.gorm.com.tr } \\
\text { e- mail: info@gorm.com.tr }\end{array}$ \\
\cline { 2 - 3 } & DOI:10.201613/GORM.2017.711 \\
\hline
\end{tabular}

How to cite this article: Senturk S. Kagitci M. Guvendag Guven ES. Thrombocyte Alterations in Pregnant Women with Gestational Diabetes Mellitus. Gynecol Obstet Reprod Med 2018;24(1):12,-16 and organ damage due to increased risk of atherosclerosis as a result of endothelial damage (1).

It is understood that changes in platelet number, function and activity in presence of diabetes mellitus have an important role in development of endothelial damage and increased risk of cardiovascular disease according to recent studies (2). Efficiency of platelet function and activity changes for prediction of prognosis in inflammatory diseases (e.g. Crohn's disease, myocardial infarction, DM.) is investigated in recent years $(3,4)$. It has been shown in some studies that mean platelet volume (MPV), plateletcrit (PCT) and platelet distribution width (PDW) which have a role to show the activity and functions of platelets have important roles in prediction of D.M. complications.

No consensus is established for glucose amount that should be used in oral glucose tolerance test (OGTT) for screening of pregnant women. 50gr OGTT is used for screening in low risk pregnancies in many clinics, however, in case of obesity, glycosuria, history of gestational diabetes mellitus (GDM) and macrosomic baby and DM. in the family $100 \mathrm{gr}$ oral glucose tolerance test is preferred. GDM develops in the second period of the pregnancy which is called the catabolic period in which the energy need is increased for fetal growth. Hormones that are against insulin such as human placental lactogen, cortisole, estrogen, and prolactin are secreted in this 
catabolic period. This state is compensated in normal pregnancies, in pregnancies that it is not compensated carbohydrate metabolism is impaired and gestational diabetes mellitus is seen (5). There are many studies showing that atherosclerotic cardiovascular disease risk is increased in presence of GDM and platelet function and activity might play an important role. It is thought that platelets contribute to development of atherosclerotic complications due to their role in inflammation and endothelial damage development $(7,8)$.

Efficiency of MPV, PDW and PCT, that have role in evaluating platelet function and activity, in prediction of complications of atherosclerotic cardiac diseases in prediabetic and diabetic patients is tried to be show in recent years. In many studies, increase of MPV in presence of D.M. is detected and its relation to development of cardiovascular diseases, nephropathy and retinopathy is reported $(9,10)$. Generally, changes in MPV values are parallel to changes in PDW values. The reason for simultaneous increase of MPV and PDW in cases with GDM is thought to be the same (11).

Mean platelet volume, PDW and PCT values are thought to be important in prediction of prognosis and complications of diseases that have inflammation in ethiopathogenesis and the importance is tried to be shown with studies recently.

The purpose of our study is to show the changes of number, morphology and functions of platelets in presence of GDM comparing to patients that are not diagnosed with GDM. We investigated the changes in MPV, PDW and PCT values in cases that we diagnosed with GDM in our study.

\section{Material and Method}

The study was conducted in Recep Tayyip Erdoğan University Research and Training Hospital Obstetrics and Gynecology Clinic between 31.08.2011 and 31.08.2014 with the permission of ethical committee. 928 cases aged 15-47 at 26-28 weeks of gestations had been included in the study. Each case included in the study was informed about the study and included voluntarily.

Venous blood was collected in appropriate circumstances from all cases included in the study following 8-10 hours of fasting for platelet count and $75 \mathrm{gr}$ oral glucose tolerance test was performed afterwards. Cases with previously diagnosed iron deficiency anemia, vitamin B12 deficiency, folic acid deficiency, and acute and chronic inflammation were excluded as platelet count, MPV measurement and PCT values will be affected negatively. Cases with Hemoglobin value below $12 \mathrm{gr} / \mathrm{dL}$, known or previous idiopathic thrombocytopenic purpura and thrombocytosis and cases with collagen tissue disease were not included in the study. Cases that had blood glucose levels over $126 \mathrm{gr} / \mathrm{dL}$ following 8-10hours of fasting from venous blood and over $200 \mathrm{gr} / \mathrm{dL}$ at any hour of the day were not included as these cases are diagnosed with diabetes melli- tus according to ADA criteria (12). Patients with history of drugs such as antibiotics, hormone replacement and anti-hypertensive drugs (e.g. thiazides) were not included in the study as these drugs might interfere with OGTT evaluation.

Patients were recommended a diet containing 150gr/day carbohydrates and no restriction of physical activity three days prior to the test for true evaluation of OGTT. Venous blood was collected from all cases to evaluate the fasting blood glucose levels after venous blood collection for platelet count and 10 minutes prior to $75 \mathrm{gr}$ OGTT. OGTT was performed while patients were in sitting position and $75 \mathrm{gr} / 100 \mathrm{~mL}$ glucose solution had been administered to all cases. Blood glucose levels were evaluated wit Abboot Architect device using standard methods.

Oral glucose tolerance test were evaluated according to ADA (American Diabetes Association) criteria. Fasting glucose level $\geq 92 \mathrm{mg} / \mathrm{dL}, 1^{\text {st }}$ hour blood glucose level $\geq 180 \mathrm{mg} / \mathrm{dL}$ and $2^{\text {nd }}$ hour blood glucose level $\geq 153 \mathrm{mg} / \mathrm{dL}$ had been accepted as pathological and the patients were diagnosed as GDM in case any pathological value is detected (12).

Venous blood collected from all patients was put in tubes containing EDTA for platelet measurement evaluation and measurements were done in 3 hours without hemolysis. Venous blood is centrifuged around 7-10 minutes and modern hematology analyzer electrical impedance technique is used for correct measurement of MPV, PCT and PDW (Abbot, Cell Dyn Ruby). Normal values were accepted as $100.000 \mathrm{~mm} 3 / \mathrm{L}-$ $300.000 \mathrm{~mm}^{3} / \mathrm{L}$ for platelet count, $<0.1 \mathrm{ng} / \mathrm{mL}$ for PCT, $9-14 \mathrm{fl}$ for PDW.

\section{Statistical Evaluation}

NCSS (Number Cruncher Statistical System) 2007\& PASS (Power Analysis and Sample Size) 2008 Statistical Software (Utah, USA) program was used for statistical analysis. Descriptive statistical methods (mean, standard deviation, frequency, and ratio, minimum, maximum) were used along with student $t$ test for comparison of two groups of variables with normal distribution for evaluation of quantitative data. Significance was evaluated at $\mathrm{p}<0.01$ and $\mathrm{p}<0.05$ values.

\section{Results}

The age of the included cases ranged between 15 to 47 years and the median age was $29.10 \pm 5.74$ years. Platelet count ranged between $76000 \mu \mathrm{m} / \mathrm{L}$ and $488000 \mu \mathrm{m} / \mathrm{L}$ and the mean platelet count (Plt) was $237,18 \pm 57,82 \mu \mathrm{m} / \mathrm{L}$. Mean platelet volume (MPV), plateletcrit (PCT) and platelet distribution width (PDW) were evaluated in all cases. MPV ranged from 4.82 to $13.60 \mathrm{fl}$ and mean MPV value was $7.55 \pm 1.31 \mathrm{fl}$; PCT ranged from 0.078 to $0.339 \mathrm{fl}$ and the mean $\mathrm{PCT}$ was $0.17 \pm 0.04 \mathrm{fl}$; PDW ranged from 17.4 to $24.2 \mathrm{ng} / \mathrm{L}$ and the mean was $20.01 \pm 1.06 \mathrm{ng} / \mathrm{ml}$. Distribution of definition features shown at table 1 . 
Table 1: Distribution of Definition Features

\begin{tabular}{|c|c|c|c|}
\hline & & Minimum-Maximum & Mean \pm SS \\
\hline \multicolumn{2}{|l|}{ Age (year) } & $15-47$ & $29.10 \pm 5.74$ \\
\hline \multicolumn{2}{|c|}{ Thrombocyte (103 per $\mu \mathrm{L})$} & $76-488$ & $237.18 \pm 57.82$ \\
\hline \multicolumn{2}{|c|}{ Mean Platelet Volume (fL) } & $4.82-13.60$ & $7.55 \pm 1.31$ \\
\hline \multicolumn{2}{|c|}{ Plateletcrit (fL) } & $0.078-0.339$ & $0.17 \pm 0.04$ \\
\hline \multirow{2}{*}{\multicolumn{2}{|c|}{ Platelet Distribution Width (ng/mL) }} & $17.4-24.2$ & $20.01 \pm 1.06$ \\
\hline & & $\mathbf{n}$ & $\%$ \\
\hline Gestational & Non-diabetes mellitus & 737 & 79.4 \\
\hline Gestational & Diabetes mellitus & 191 & 20.6 \\
\hline
\end{tabular}

In the study group,79.4\% $(\mathrm{n}=737)$ of the cases had no GDM and $20.6 \%(n=191)$ of the cases were diagnosed as GDM. Distribution of GDM shown at figure 1.

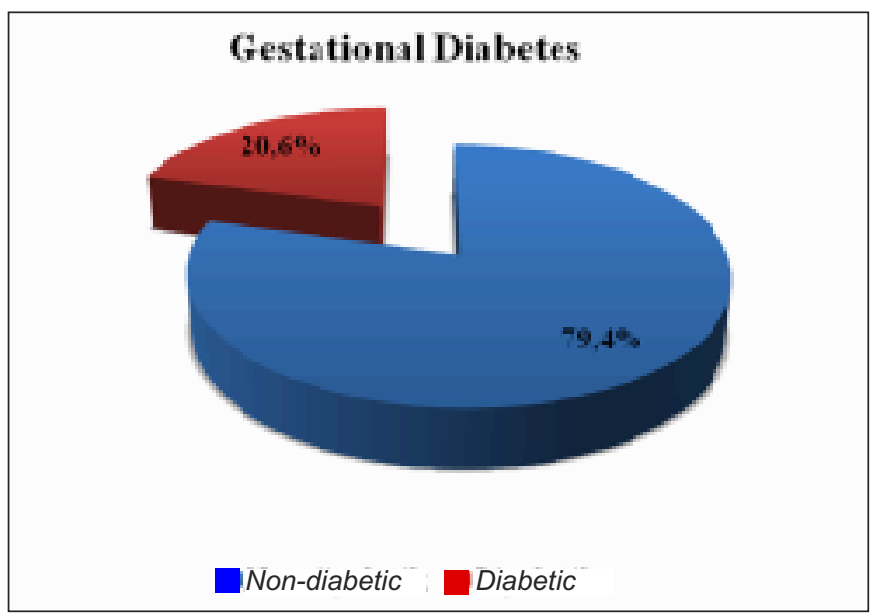

Figure 1: Distribution of gestational diabetes mellitus

The mean age of the cases with GDM had significantly higher than the cases that are not diagnosed with GDM. ( $p=0.001 ; p<0.01)$. Plt value of cases with gestational diabetes mellitus was significantly higher than the cases without GDM $(p=0.022 ; p<0.05)$. Mean MPV and PDW values were not different at a statistically significant level between the cases with GDM and the cases without GDM $(p>0.05)$. Mean PCT value of GDM cases were significantly higher than the cases without GDM ( $p=0.001 ; p<0.01)$. Evaluation of Descriptive Data in Relation to Frequency of GDM shown at table 2.

\section{Discussion}

World Health Organization (WHO) reported that cardiovascular diseases are responsible for $30 \%$ of mortality all around the world. Sedentary lifestyle, obesity, increased frequency of DM. is counted among important reasons of increased frequency of cardiovascular diseases (13). Frequency of hypertension and cardiovascular diseases is higher with diabetes mellitus diagnosis compared to the normal population. Hormones are protective for females at reproductive ages; however, cardiovascular disease risk is increased with diseases such as polycystic ovary, GDM developing during these ages (14).

Gestational diabetes mellitus plays an important role in development of especially atherosclerotic cardiovascular diseases by causing DM. type 2 in postpartum period in addition to increased risk of atherosclerosis during pregnancy. It is reported that DM. might develop at a rate of $2.6-70 \%$ in postpartum 6 weeks to 28 years' period in GDM cases. GDM is an important health problem as it increases maternal and fetal morbidity and mortality and also the risk of postpartum D.M. development $(15,16)$.

We have aimed to show the influence of platelet function and activity in GDM cases as it is known that insulin resistance and impaired glucose tolerance causes secretion of inflammatory mediators (CRP, visfatin, omentin, IL-6 etc.) and changes in platelet function and activity starts endothelial damage (17).

Table 2: Evaluation of descriptive data in relation to frequency of gestational diabetes mellitus

\begin{tabular}{lccc}
\hline & Mean \pm SS & Mean \pm SS \\
& Gestational diabetes mellitus & Gestational non-diabetes mellitus \\
$(\mathrm{n}=191)$ & $p$ & $0.001^{* *}$ \\
\hline Age (year) & $31.75 \pm 5.42$ & $28.42 \pm 5.62$ & $0.022^{*}$ \\
Thrombocyte (103 per $\mathrm{HL})$ & $245.69 \pm 60.42$ & $234.98 \pm 56.96$ & 0.132 \\
Mean Platelet Volume (fl) & $7.68 \pm 1.33$ & $7.52 \pm 1.30$ & $0.001^{* *}$ \\
Plateletcrit (fl) & $0.18 \pm 0.04$ & $0.17 \pm 0.04$ & 0.590 \\
Platelet Distribution Width $(\mathrm{ng} / \mathrm{mL})$ & $20.04 \pm 1.05$ & $20.00 \pm 1.07$ & \\
\hline
\end{tabular}


One or two step approach could be use for the diagnosis of the D.M or GDM. In the two step approach, 50 gr glucose is given to the patients and blood glucose is measured after an hour and if it is over $140 \mathrm{mg} / \mathrm{dL} 75$ or $100 \mathrm{gr}$ OGTT is performed for diagnosis. However, no consensus is established for the diagnosis of GDM and further wider research is recommended. We have diagnosed our cases with GDM following $75 \mathrm{gr}$ oral glucose tolerance test.

We have detected that mean maternal age is higher in cases diagnosed with GDM compared to cases that are not diagnosed with GDM in our study. Results obtained from many studies showed that obesity, insulin resistance, and increased maternal age are important risk factors for development of GDM (14). Maternal age could be a risk factor for GDM and therefore contribute to development of atherosclerotic vascular and organ damage according to data obtained from our study.

It was shown that tendency to hypercoagulability and homeostasis disorders during pregnancy could be related to development of atherosclerosis and ischemic cardiovascular diseases (11). High and low levels of platelet count are important causes of coagulation disorders. We have aimed to show the relation between coagulopathy and GDM by evaluation of platelet numbers. Platelet measurements were in between the normal ranges in all cases; however, platelet numbers were insignificantly higher in cases with GDM. We think that higher number of GDM cases included in the study with regard to clinical and demographical characteristics of the patients would cause different results and we would still find platelet count of GDM cases higher.

We have found that MPV value of cases with or without GDM were in between the normal ranges and there was no difference between the groups. MPV is an indicator of platelet functions and also increased number of immature platelets in the bone marrow. Therefore, we concluded that MPV measurements in the normal limits should be parallel to platelet production and count.

Platelet distribution width values were increased in both groups with and without GDM however measurements were not different between the groups in our study. Increased PDW values in our study groups could be related to hypercoagulopathy, inflammation developing during pregnancy and increased atherosclerosis risk. PCT values were increased in both groups, and it was significantly higher in the GDM group compared to the other group. In some studies, it was detected that PCT values were higher in preterm deliveries and repeated abortions and frequency of preeclampsia, GDM and hypertension were increased (18). It was reported that preeclampsia, hypertension and GDM presence are important reasons for atherosclerotic vascular and organ damage and therefore PCT measurements might be helpful for prediction of atherosclerosis. Higher values of PCT for both groups in our study could be explained as the pregnancy itself are a con- dition that could be related to atherosclerosis, inflammation and coagulopathy. Higher values of PCT in GDM cases could be explained by they are a group with higher risk for atherosclerosis.

In summary, the place of platelets for prediction of diagnosis or prognosis of atherosclerosis and inflammation for the diseases with and ethiopathogenesis of atherosclerosis. Data obtained from many studies had shown that platelet function and activity might be meaningful for diagnosis and prognosis prediction. However, general idea is that the role of MPV, PDW and PCT measurements should be investigated with wider studies including more patients with regard to demographical and clinical characteristics of the patients.

\section{Conclusion}

There is tendency to inflammation in GDM and platelets are part of inflamatuary process in human metabolism. In addition, evaluation of platelet number, function and activity could be helpful for prediction of atherosclerotic vascular and organ damage in cases diagnosed with gestational diabetes mellitus. Our findings show that, platelets are affected in GDM. We believe that further prospective studies with large sample size in this subject will help understanding of pathophysiology of platelet alterations in GDM.

: The authors declare that there is no conflict of interests regarding the publication of this paper.

\section{References}

1. Creager MA, Lüscher TF, Cosentino F and Beckman JA. Diabetes and vascular disease: pathophysiology, clinical consequences, and medical therapy: Part I. Circulation 2003 Sep 23;108(12):1527-32. DOI:10.1161/01.CIR. 0000091257.27563 .32

2. Ferreiro JL, Joan A GH and Dominick JA. Platelet abnormalities in diabetes mellitus. Diabetes \& Vascular Disease Research 2010;7(4):251-9. doi: 10.1177/14791641103 83994

3. Kodiatte TA, Manikyam UK, Rao SB, Thej MJ, Madhavi R, Harendra KML et al. Mean Platelet Volume in Type 2 Diabetes Mellitus. J Lab Physicians 2012 Jan-Jun;4(1):59. doi:10.4103/0974-2727.98662

4. Chang HA, Hwang HS, Park HK, Chun MY, Sung JY. The Role of Mean Platelet Volume as a Pre-dicting Factor of Asymptomatic Coronary Artery Disease. Korean J Fam Med2010;31(8):600-6. https://doi.org/10.4082/kjfm.2010. 31.8 .600

5. Lee AJ, Hiscock RJ, Wein P, Walker SP, Permezel M. Gestational diabetes mellitus: Clinical predictors and long-term risk of developing type 2 diabetes: a retrospective cohort study using survival analysis. Diabetes Care 2007 Apr;30(4):878-83. https://doi.org/10.2337/dc061816 
6. Harreiter J, Dovjak G, Kautzky-Willer A. Gestational diabetes mellitus and cardiovascular risk after pregnancy. Womens Health (Lond) 2014 Jan;10(1):91-108. doi: 10.2217/whe. 13.69

7. Gioia S, Cerekja A, Larciprete G, Vallone C, Demaliaj E, Evangelista MT, et al. Gestational diabetes: is it linked to platelets hyperactivity? Platelets 2009;20(2):140-1. doi: 10.1080/09537100802630062

8. Stein PD, Goldman J, Matta F, Yaekoub AY. Diabetes mellitus and risk of venous thromboembolism. Am J Med Sci 2009;337(4):259-64.doi:10.1097/MAJ.0b013e31818bbb $8 \mathrm{~b}$

9. Coban E, Bostan F, Ozdogan M. The mean platelet volume in subjects with impaired fasting glucose. Platelets 2006;17(1):67-9. Erratum in: Platelets 2006; 17:350. DOI: 10.1080/09537100500220729

10. Dundar O, Yoruk P, Tutuncu L, Erikci AA, Muhcu M, Ergur AR, et al. Longitudinal study of platelet size changes in gestation and predictive power of elevated MPV in development of pre-eclampsia. Prenat Diagn 2008;28(11):1052-6. doi: 10.1002/pd.2126

11. Piazze J, Gioia S, Spagnuolo A, Cerekja A. Platelets in pregnancy. Journal of Prenatal Medicine 2011 Oct;5 (4): 90-2.

12. American Diabetes Association Standards of Medical Care in Diabetes. Classification and diagnosis of diabetes. Diabetes Care 2017; 40 (Suppl. 1): S11-S24
13. WHO. Global Status Report on Noncommunicable Diseases 2010. WHO Press, Geneva, Switzerland 2011.

14. Kautzky-Willer A, Dorner T, Jensby A, Rieder A. Women show a closer association between educational level and hypertension or diabetes mellitus than males: a secondary analysis from the Austrian HIS. BMC Public Health 2012 May 30;12:392. doi: 10.1186/1471-2458-12-392

15. Kim C, Newton KM, Knopp RH. Gestational diabetes and the incidence of Type 2 diabetes: a systematic review. Diabetes Care 2002 Oct;25(10):1862-8. https://doi.org/ $10.2337 /$ diacare.25.10.1862

16. Bozkurt L, Göbl CS, Tura A, Marek C, Thomas P, Lana K, et al. Fatty liver index predicts further metabolic deteriorations in women with previous gestational diabetes. PLoS One 2012;7(2): e32710. https://doi.org/10.1371/ journal.pone. 0032710

17. Legato MJ, Gelzer A, Goland R, Ebner SA, Rajan S, Villagra V, et al. Gender-specific care of the patient with diabetes: review and recommendations. Gend. Med 2006;3(2):131-58. https://doi.org/ 10.1016/S1550-8579 (06)80202-0

18. Thangaratinam S, Rogozinska E, Jolly K, Glinkowski S, Roseboom T, Tomlinson JW et al. Effects of interventions in pregnancy on maternal weight and obstetric outcomes: meta-analysis of randomised evidence. BMJ 2012 May 16;344: e2088. doi: https://doi.org/10.1136/bmj.e2088 